\title{
COMUNICAÇÃO BREVE O transtorno bipolar como experiência: a perspectiva dos filhos
}

\author{
Bipolar disorder as experience: the perspective of children
}

Lia Keuchguerian Silveira Campos', Flávia Machado Seidinger-Leibovitz', Amilton dos Santos Junior², Egberto Ribeiro Turato ${ }^{2}$

\section{RESUMO}

Objetivo: Compreender o transtorno afetivo bipolar (TAB) e suas implicações pela perspectiva dos filhos adultos de mães que receberam o diagnóstico quando eles eram crianças. Métodos: Pesquisa qualitativa, por meio de entrevistas semiestruturadas de questões abertas em profundidade com 21 filhos de pacientes do Ambulatório de Psiquiatria Geral de Adultos do HC/Unicamp. A técnica de tratamento de dados foi feita por meio da análise de conteúdo das entrevistas transcritas na íntegra e categorização. Resultados: Os significados psicológicos atribuídos pelos filhos à experiência de ter uma mãe com TAB possibilitaram a elaboração de um esquema da infância, adolescência à vida adulta. Os achados revelam que na infância a instabilidade da mãe, característica do TAB, gera sentimentos de culpa e desamparo nos filhos, pela percepção da introspecção materna nos períodos dos episódios em contraste com os momentos de remissão, nos quais percebem envolvimento emocional e disponibilidade para a maternidade. Na adolescência, os filhos contestam as demandas de suas mães. Demonstram identificação com os sintomas maternos. Na vida adulta, há percepção de melhora na relação mãe-filho diante da abdicação das escolhas pessoais para a dedicação aos cuidados à mãe. Os achados revelam que a compreensão do TAB feita por meio da experiência dos filhos com suas mães fica apenas na racionabilidade, pois eles não conseguem manejá-la de forma efetiva e sentem-se aprisionados. Conclusões: A equipe de saúde pode auxiliar os filhos desde o manejo de questões cotidianas até o diagnóstico precoce de psicopatologias ao se dedicar ao acolhimento deles quando da vinda de suas mães ao tratamento.

\section{ABSTRACT}

Objective: To understand bipolar disorder (BD) from the perspective of the adult offspring of mothers who received the diagnosis when they were children and their implications. Methods: Qualitative research conducted through in-depth semistructured interviews with 21 children of patients of the General Psychiatry Outpatient Clinic for Adults at HC/ Unicamp. The technical data processing was done through qualitative content analysis of the transcribed interviews and categorization. Results: The psychological meanings attributed by the offspring to the experience of having a mother with $\mathrm{BD}$ enabled the elaboration of a schematic from childhood, adolescence to adulthood. The findings reveal that in childhood

1 Universidade Estadual de Campinas, Faculdade de Ciências Médicas (Unicamp/FCM); Unicamp/FCM, Laboratório de Pesquisa Clínico-Qualitativa (LPCQ), Departamento de Psicologia Médica e Psiquiatria da Faculdade de Ciências Médicas (DPMP).

Recebido em

21/3/2017

Aprovado em 2 Universidade Estadual de Campinas, Faculdade de Ciências Médicas (Unicamp/FCM), Laboratório de Pesquisa Clínico-Qualitativa (LPCQ), Departamento de Psicologia Médica e Psiquiatria da Faculdade de Ciências Médicas (DPMP). 


\section{Keywords}

Bipolar disorder, mother child relations, family relations, user embracement, qualitative research. the instability of the mother, characteristic of $\mathrm{BD}$, generates feelings of guilt and helplessness in the children, by the perception of maternal introspection in the periods of the episodes in contrast to moments of remission in which they perceive emotional involvement and availability for motherhood. In adolescence, the children challenge the demands of their mothers. Demonstrate identification with maternal symptoms. In adulthood, there is perception of improvement in the mother-child relationship due to the abdication of personal choices for dedication to the mother's care. The findings reveal that the understanding of $\mathrm{BD}$ made through the experience of the offspring with their mothers is only in rationality, they can not handle it effectively and they feel trapped. Conclusions: The health team involved can help these children from management of daily issues to the early diagnosis of psychopathologies by dedicating themselves to receiving these children when their mothers come to the treatment.

\section{INTRODUÇÃO}

As implicações dos aspectos genéticos e ambientais em filhos(as) de mães com transtorno afetivo bipolar (TAB) têm sido amplamente discutidas, com achados que enfatizam tratar-se de uma população de risco para psicopatologias e dificuldades psicossociais ${ }^{1,2}$. Filhos de pacientes com TAB apresentam maiores taxas de transtornos de humor, de ansiedade, de déficit de atenção e hiperatividade e de comportamento disruptivo que proles de pacientes com outros transtornos psiquiátricos ou de controles saudáveis ${ }^{3}$. Assim, os filhos compõem o grupo de estudo ideal para avaliar a história natural da doença e seus sintomas prodrômicos ${ }^{3}$.

Estudos sobre as experiências subjetivas do conviver com uma mãe com transtornos mentais são, todavia, escas$\operatorname{sos}^{4,5}$. Entende-se que muitos são os motivos para se estudar os filhos de mulheres com TAB. A presente pesquisa visa compreender as implicações do transtorno a partir da perspectiva desses indivíduos.

\section{MÉTODOS}

Utilizou-se o método qualitativo aplicado à área da saúde ${ }^{6}$, por não se tratar de investigação sobre relações de causalidade, mas por procurar identificar as complexas interações de fatores envolvidas ${ }^{7}$ e compreender vivências, sentimentos, ideias e comportamentos dos sujeitos estudados ${ }^{8}$.

Os dados foram coletados por meio de entrevistas semiestruturadas, com questões abertas em profundidade, após assinatura do Termo de Consentimento Livre e Esclarecido. A amostra (Tabela 1) foi intencional e fechada pelo critério de saturação. Os participantes da pesquisa foram filhos(as) adultos(as) de mães atendidas no Ambulatório de Psiquiatria Geral de Adultos do Hospital de Clínicas da Universidade Estadual de Campinas (HC/Unicamp) que receberam o diagnóstico de TAB quando os sujeitos do estudo eram crianças.
A técnica de tratamento de dados consistiu da análise de conteúdo ${ }^{9}$ do conjunto do material das gravações das entrevistas transcritas na íntegra, para categorização segundo núcleos de significado emergentes das falas, em um rigoroso processo de reflexão e associações com teorias já publicadas na literatura. As categorias relatadas neste artigo passaram por validação externa com especialistas, além da supervisão por pares acadêmicos do Laboratório de Pesquisa Clínico-Qualitativa da Unicamp.

O estudo foi aprovado pelo Comitê de Ética em Pesquisa da Faculdade de Ciências Médicas da Unicamp (CAAE: 12593713.7.0000.5404).

\section{RESULTADOS}

No processo de interpretação dos dados, foi escolhido apresentar um esquema formado por meio da ressignificação das experiências emocionais da infância, adolescência e vida adulta feita pelos filhos(as) para descrever como entendem e vivenciam o TAB de suas mães e suas implicações. Tais categorias emergiram da compreensão de que a percepção do TAB e os comportamentos subsequentes sofriam influências das características particulares de cada uma dessas fases da vida desses indivíduos.

\section{Categoria 1: Infância}

Os entrevistados referem recordações da infância permeadas pela insegurança na relação com suas mães e a percepção de que havia algo com que elas próprias não conseguiam lidar e que eles se sentiam culpados e impelidos a se adaptar. Essa insegurança se dava pela instabilidade característica do quadro psiquiátrico e também pela apreensão rudimentar de que elas ora pareciam envolvidas emocionalmente, ora introspectivas, com desligamento emocional dos filhos. Contam sobre um receio em relação a poderem se mostrar espontâneos ou terem que se apresentar apenas obedientes. 
Tabela 1. Caracterização da amostra segundo idade, gênero, situação conjugal e ocupação atual

\begin{tabular}{|c|c|c|c|c|}
\hline Participante 01: & 25 anos, & mulher, & noiva, & funcionária pública. \\
\hline Participante 02: & 40 anos, & mulher, & casada, & do lar. \\
\hline Participante 03: & 31 anos, & mulher, & casada, & estudante. \\
\hline Participante 04: & 20 anos, & mulher, & solteira, & do lar. \\
\hline Participante 05: & 22 anos, & homem, & noivo, & tecnólogo. \\
\hline Participante 06: & 36 anos, & mulher, & casada, & do lar. \\
\hline Participante 07: & 43 anos, & mulher, & casada, & vendedora. \\
\hline Participante 08: & 32 anos, & homem, & casado, & profissional de logística. \\
\hline Participante 09: & 18 anos, & mulher, & solteira, & do lar. \\
\hline Participante 10: & 35 anos, & mulher, & casada, & profissional de logística. \\
\hline Participante 11: & 18 anos, & mulher, & solteira, & estudante. \\
\hline Participante 12: & 18 anos, & mulher, & solteira, & estudante. \\
\hline Participante 13: & 29 anos, & mulher, & solteira, & advogada. \\
\hline Participante 14: & 19 anos, & mulher, & solteira, & estudante. \\
\hline Participante 15: & 28 anos, & homem, & solteiro, & mecânico. \\
\hline Participante 16: & 34 anos, & mulher, & casada, & contadora. \\
\hline Participante 17: & 38 anos, & homem, & casado, & pedreiro. \\
\hline Participante 18: & 25 anos, & homem, & solteiro, & estudante. \\
\hline Participante 19: & 22 anos, & homem, & casado, & motorista. \\
\hline Participante 20: & 42 anos, & homem, & casado, & técnico em enfermagem. \\
\hline Participante 21: & 23 anos, & homem, & solteiro, & desempregado. \\
\hline
\end{tabular}

“(..) sempre fui muito preocupada. Quando notava que ela estava um pouco esquisita, um pouco estranha de comportamento, eu já percebia. Eu era a primeira que percebia, mesmo sendo criança, já tinha isso." (P1)

Os filhos relatam preocupação precoce quanto à capacidade das mães de entenderem as intenções das pessoas que as cercam e de potenciais reações agressivas.

"Quando ela estava em crise... Aí que era pior, porque ela ficava agressiva, batia na gente, não reconhecia que a gente era filha dela. Nossa, foi terrível mesmo..." (P7)

Também foi relatada a percepção de momentos em que as mães ficavam ensimesmadas, sendo impossível acessá-las, e as apreensões decorrentes dessas ocasiões.

“(...) eu tinha que viver atrás da minha mãe vinte e quatro horas. Para mim, qualquer segundo longe, eu achava que ela ia morrer." (P14)

Em um processo de ressignificação, os participantes relatam que apenas na vida adulta perceberam o quanto foram depositários das angústias maternas. Sentiam-se, inclusive, culpados por suas alterações de humor.

"A vida inteira eu cresci ouvindo, porque tudo desencadeou depois que eu nasci. Foi no parto (...) cresci ouvindo que sou a culpada (...). Por outro lado, sou a única, no momento de crise, que ela aceita (...) tipo: o motivo e a cura sabe, a causa e a cura." (P16)

Os filhos relatam que a instabilidade é a característica mais marcante do quadro de suas mães, com envolvimento e disponibilidade em períodos de remissão, mas gerando neles sentimentos de desamparo durante os episódios. Sobre a necessidade de cuidado a elas devido ao TAB, contam sobre um amadurecimento precoce sustentado pelo sentimento de culpa, como se eles precisassem cuidar de suas mães para reparar o que pensam que fizeram com elas.

\section{Categoria 2: Adolescência}

Na adolescência, os filhos identificam em suas mães movimentos de rejeição, que agora percebem associados ao quadro clínico. Não referem sentir mais culpa, porém vergonha diante do quadro clínico materno. 
"Estou falando isso agora com certa maturidade, mas na época [da adolescência], era difícil entender. Não queria saber da minha mãe, pelo amor de Deus ela era louca, tinha raiva dela entendeu!" (P8)

As demandas de suas mães são sentidas como invasivas. Aparecem movimentos reativos, com a finalidade de se contraporem ao que identificam como projeções de ciúmes e competição.

"Adolescência acho que foi a parte pior da doença dela, ela era muito agressiva (...). Até hoje, nunca tive oportunidade de conversar com o médico (...) ela tentava me imitar, nas crises dela, então ela queria vestir... Pegar as minhas roupas para vestir... E ela ficava às vezes com... Eu percebia... com ciúmes ou raiva de mim junto com o meu pai, não sei o que ela imaginava que era..." (P6)

Os movimentos reativos são acompanhados de agressividade comparável àquela das mães nos conflitos presenciados na infância. A percepção da semelhança entre seus comportamentos de adolescentes e os episódios de suas mães faz com que sintam identificação com os sintomas maternos.

“(...) na época da adolescência, a gente achava que a nossa mãe era louca. (...) sair com ela na rua, puxa vida! Puxado! Às vezes ela não se cuidava, não arrumava o cabelo, não colocava uma roupa, ou queria colocar uma roupa indecente para a idade (...). Então a gente ficava... É o rebu da adolescência (...). Várias vezes ela saiu com camisa de força, na ambulância, sem conhecimento do problema me sentia envergonhado (...). Ela não estava conseguindo entender a cabeça dela. Você não sabe se ela está bem, porque ela oscilava de amor e raiva." (P8)

Relatam que, envergonhados, buscam isolamento social e acabam por renunciar à adolescência.

"(...) adolescência, nem sei o que é isso! Eu tive que ser... adulta nova!" (P4)

\section{Categoria 3: Vida adulta}

Na vida adulta, os dados sugerem a percepção de melhora na relação mãe-filho ao abdicarem de escolhas pessoais para se dedicarem aos cuidados com a mãe. Os participantes referem a criação de estratégias para prever os episódios, administrar medicação e poder lidar com o TAB, invertendo-se os papéis de provisão de cuidados.
“(..) a gente sempre fica em torno dela (...) tem que ter bastante paciência, autocontrole, porque senão é difícil. Às vezes eu quero fazer coisas, tomar decisões para o futuro próximo para minha vida, só que eu não posso fazer por conta dela." (P15)

"(...) acabo sendo mais mãe dela do que ela minha mãe... querendo sempre o bem-estar dela... procuro não a deixar nervosa, porque quando ela tem muitas emoções, sejam boas ou ruins, desencadeia a crise. Então, procuro levar o mínimo de preocupação possível para ela." (P13)

Percebem suas mães progressivamente dependentes de seus cuidados. Sentem-se sobrecarregados, mas também recompensados pela proximidade e pelo aprendizado pessoal.

"(...) antes eu via muito negativo, mas hoje acho que tem um lado positivo... eu sou quem eu sou hoje, porque tive esta experiência. Não daria valor para as coisas que dou hoje, se não tivesse passado por isso. Hoje eu dou valor para as coisas bem mais simples, um dia em paz, um dia quando ela está bem... eu dou valor já nesse dia." (P1)

Os entrevistados relatam que suas mães demonstram uma afinidade com a morte:

"(...) ela pegou chumbinho e colocou na comida (...) a intenção dela era nós três morrermos juntos." (P4)

"... tenho uma cicatriz... minha mãe tentou me ma$\operatorname{tar}($...) Foi quando eu nasci. Ela não me aceitava. Estava numa crise e tentou me matar com uma facada, oito meses de idade." (P5)

Os filhos referem que o tema da morte faz parte de suas vidas e o associam ao transtorno. Colocam-se, porém, como responsáveis pela manutenção da vida de suas mães, o que se torna mais um motivo para lhes dedicar cuidados.

\section{DISCUSSÃO}

Os achados revelam a perspectiva dos filhos acerca do $T A B$ e as implicações em suas vidas, destacando especificidades da relação mãe-filho, inclusive análogas aos achados de Méndez et al..$^{10}$ sobre a percepção da presença da enfermidade materna desde muito cedo e sentimentos de culpa.

A percepção dos filhos de que há algo com que suas mães não conseguem lidar e que eles precisam se adaptar 
mostra-se relacionada ao que foi descrito no estudo de Bin et al."1" sobre a tendência do paciente com TAB de apresentar intensa autocrítica, geradora de angústia e possível de ser projetada nas pessoas com quem convivem. Dessa forma, aos filhos resta uma postura obediente, a fim de evitar conflitos. Como depositários dessas projeções, sentem-se também cobrados ao amadurecimento precoce e rejeitados em seus atos espontâneos.

Segundo Tonelli'12, o prejuízo na percepção de estados mentais compromete as habilidades dos pacientes com TAB de estabelecerem relacionamentos interpessoais saudáveis, gerando tensão repercutindo no quadro clínico. Pode-se pensar que os filhos percebem essas limitações de suas mães e procuram lidar com esse aspecto de forma a poupá-la, adaptando-se a essa condição na infância e rebelando-se contra ela na adolescência, motivados pelo peculiar sentimento de incompreensão dessa fase.

De acordo com Maoz et al. ${ }^{13}$, a agressividade é uma característica marcante em pré-escolares filhos de pais com TAB. Os achados da presente pesquisa apontam para agressividade também na adolescência, o que corrobora Narayan et al. ${ }^{14}$. Para esses autores, as mães com TAB cometiam violência interparental, o que contribuiria para a agressividade nessa fase ${ }^{14}$.

Na vida adulta, os achados apontam para um sentimento predominante de sobrecarga, assim como foi encontrado no estudo de Bauer et $a l .^{15}$. A responsabilidade, vivenciada como um fardo, é identificada por um sofrimento específico dos filhos cuidadores como efeito psicossocial do transtorno materno. Também ocorre impacto do TAB na família e há prejuízos correlatos, enfrentados pelos filhos, no seu funcionamento no trabalho ou escola's.

Esses autores ${ }^{15}$ também identificaram a percepção de que as vivências tiveram um aspecto recompensador. Os entrevistados do presente estudo relatam experiências emocionais permeadas de desamparo e rejeição, entretanto conseguem encontrar sentido na dedicação aos cuidados com suas mães.

Os participantes referem estratégias para lidar com o transtorno, porém sentem-se sobrecarregados e frustrados. Segundo Bauer et al. ${ }^{15}$, haveria uma tendência dos filhos em utilizar formas depressivas de enfrentamento. Os achados revelam que, embora tenham compreensão em relação ao transtorno de suas mães e suas implicações, essa compreensão permanece no plano da racionalidade/intelectualidade e eles demonstram não conseguir manejá-las de forma efetiva, sentindo-se aprisionados.

A presente pesquisa sugere que os filhos de mulheres com TAB precisam ser avaliados em singularidade. Gladstone et al. ${ }^{4}$ ressaltam a importância de ver além da resiliência e também do fato de serem uma população de risco para, assim, identificar suas reais necessidades.

\section{CONCLUSÕES}

Os achados da presente pesquisa demonstram como as vivências dos filhos das mulheres com TAB podem ser complexas e demandar apoio emocional. Dessa forma, a equipe de saúde mental envolvida pode auxiliar os filhos desde o acolhimento, suporte emocional e manejo de questões cotidianas até o diagnóstico precoce de psicopatologias. Ao se dedicar a receber esses filhos, quando da vinda de suas mães ao tratamento, a equipe pode abarcar maior número de fatores envolvidos na evolução do TAB, assim como protegê-los das implicações e oferecer um tratamento mais completo.

\section{CONTRIBUIÇÕES INDIVIDUAIS}

Lia Keuchguerian Silveira Campos - Contribuiu na concepção e desenho do estudo, na análise e interpretação dos dados, na elaboração do artigo e na revisão crítica de todo o conteúdo intelectual.

Flávia Seidinger-Leibovitz - Contribuiu na análise e interpretação dos dados, na elaboração do artigo e na revisão crítica de todo o conteúdo intelectual.

Amilton dos Santos Junior - Contribuiu na análise e interpretação dos dados, na elaboração do artigo e na revisão crítica de todo o conteúdo intelectual.

Egberto Ribeiro Turato - Contribuiu na concepção e desenho do estudo, na análise e interpretação dos dados e na revisão crítica de todo o conteúdo intelectual.

A versão final foi aprovada por todos os autores

\section{CONFLITOS DE INTERESSES}

Não há conflitos de interesses.

\section{AGRADECIMENTOS}

À Coordenação de Aperfeiçoamento de Pessoal de Nível Superior (Capes), pela bolsa de doutorado, e aos professores Lilian do Rocio Borba e Roosevelt Moisés Smeke Cassorla, pela validação dos achados.

\section{REFERÊNCIAS}

1. Bella T, Goldstein T, Axelson D, Obreja M, Monk K, Hickey MB, et al. Psychosocial functioning in offspring of parents with bipolar disorder. J Affect Disord. 2011;133(1-2):204-11.

2. Birmaher B, Axelson D, Goldstein B, Monk K, Kalas C, Obreja M, et al. Psychiatric disorders in preschool offspring of parents with bipolar disorder: the Pittsburgh Bipolar Offspring Study (BIOS). Am J Psychiatry. 2010;167(3):321-30.

3. Villa EU, Briceño PG, Palacio JD, García J. [Psychopathological profiles of offspring of subjects with bipolar disorder]. Rev Colomb Psiquiatr. 2012;41(1):15-25. 
4. Gladstone BM, Boydell KM, McKeever P. Recasting research into children's experiences of parental mental illness: beyond risk and resilience. Soc Sci Med. 2006;62(10):2540-50.

5. Leverton TJ. Parental psychiatric illness: the implications for children. Curr Opin Psychiatry. 2003;16(4):395-402.

6. Pope C, Mays N, editors. Qualitative research in health care. 3th ed. Oxford: Blackwell Publishing Ltd; 2006.

7. Creswell JW. Qualitative inquiry and research design: Choosing among five traditions. 2th ed. Thousand Oaks, CA: Sage Publications; 2007.

8. Turato ER. Tratado da metodologia da pesquisa clínico-qualitativa. Construção teórico-epistemológica, discussão comparada e aplicação nas áreas da saúde e humanas. 6th ed. Petrópolis: Vozes; 2013

9. Pope C, Ziebland S, Mays N. Qualitative research in health care. Analysing qualitative data. BMJ. 2000;320(7227):114-6.
10. Méndez JA, Albornoz-Salas 0, Pérez-OImos I. Percepción de la enfermedad mental materna por los niños. Rev Colomb Psiquiatr. 2010;39(1):110-33.

11. Bin LCP, Campos LKS, Santos Jr. A, Turato ER. Significados dos episódios maníacos para pacientes com transtorno bipolar em remissão: um estudo qualitativo. J Bras Psiquiatr. 2014;63(2):142-8

12. Tonelli HA. Empatia no transtorno afetivo bipolar. Rev Psiquiatr Clín. 2011;38(5):207-8.

13. Maoz H, Goldstein T, Axelson DA, Goldstein BI, Fan J, Hickey MB, et al. Dimensional psychopathology in preschool offspring of parents with bipolar disorder. J Child Psychol Psychiatry. 2014;55(2):144-53.

14. Narayan AJ, Chen M, Martinez PP, Gold PW, Klimes-Dougan B. Interparental violence and maternal mood disorders as predictors of adolescent physical aggression within the family. Aggress Behav. 2015;41(3):253-66.

15. Bauer R, Spiessl H, Helmbrecht MJ. Burden, reward, and coping of adult offspring of patients with depression and bipolar disorder. Int J Bipolar Disord. 2015;3:2. 